\title{
Namenregister zu Band 72
}

Die îett gedruckten Zahlen bedeuten Eigenarbeiten.

Albrich 53 Altschul 335 Ambard 216 Amsler 88, 217 Aron 318 Arruga 228 Ascher 72, 76, 79

Aubaret 209 Aubineau 211 Axenfeld 256 (P.)

B

Baillart 218

Baldino 228

Bar 58

Bartels 311, 324, 325

Baudot 228

Baurmann 103, 114

Beauvieux 228 Becker 62 Berens 217 Berneaud 323 Bessemans 332 Best 109 Betsch 336 Bier 399 Bilger 217, 250 Birch-Hirschfeldi2o,

244, 259 Blaickner 96, 265 Blonsky 245 Blumenthal 123 Boron 206 Borrel 208 Bretagne 208 Bruckner 210 Bruckner 102, 103, 114,

$4^{\circ} 3$

Caesar 319 Cange 212 Canneyt 332 Cantilo 224 Carlo 206 Chaillous 229 Charamis 209

Chennevière 209 Clausen 58, 88 Comberg 52, 107, 205 Coppez 220, 221 v. Csapody 83 Cuenod 210

$\mathrm{D}$

Danilewsky 245, 246 Decking 324 Deggeler 335 Dejean 214, 227

Namenregister zu Band 72.

411

Deutsch 230 Dieter 47 Dimissianos 213 Doumer 226 Dubar 217 Dufour 206 Dupuy-Dutemps 210 ,

212, 218 Duverger 228

E Elschnig 57 Erggelet 86, 95, 98

$\mathrm{F}$

Fabritius 62 Favory 211 Feeder 244 Feigenbaum 51, 57 Fésus 326 Feuille 227 Fischer, E.

(Basel) 369 Fischer (Leipzig) 65,

66, 75 Fleischer 57, 62 Franceschetti 113,

234, 238, 240 Frank 61 Freund 92 Friede 299

G

Gajewitsch 247 Gallois 208 Gardilicic 187 Geller 63 Genet 229 Ginzburg 59 Goldmann 334

Goldstein 331 Gonin 239 Gredsted 243 v. Grósz 328 Gruber 90 Grüter 89, 106 Guist 59, 112, 230 Gutzeit 118

$\mathrm{H}$

Hambresin 213 Hanssen 45, 324, 360 Hartinger 82, 88, 114,

319 Heesch 47 Hegner 105 Herford 121 Herrenschwand 62 Hertel 64 Herzog 316 Heß 55 
Heßberg 96, 319, 322,

3H v. Hippel 87, 248 van der Hoeve 208 vom Hofe 75, 79 Hoffmann, W. 94, 119 Hollos 329

Horniker 48 Horváth 329 Huber 336 Hudelo 219 Huneus 320

I

Igersheimer 101

Illés 330

v. Imre 101, 107, 326,

328 Ishikawa 52

J.

Jadassohn, W. 233 Jaensch 56, 60, 99, 333 Jaffé 123 Jahnke 354 Jarmersted 122 Jeß 102 Joseph 213 Jourdan 221 Jung 318 Junius 116

$\mathrm{K}$

v. Kaáli Nagy 328 Kamenetzki 61 Kantor 384 Kerenjewitsch 246 Kiranoff 58 Kleiber 96 Kobsar 246 Koby 214 Kraemer 402 Kramarewsky 244 Kraupa 59 Kreiker 327, 328, 329,

330 Kremer 318 Kretzschmar 240 Krückmann 93, 104, 258, 323 Kubik 332 Kunz 121, 244 Kyrieleis 98, no

$\mathrm{L}$

Lagrange 217 Lamache 217 Landegger 179 Larsson 59, 62, 398 Lauber 101 Lewitsky 244, 245, 246

Liebermann 327, 329 Lindner 63, 85, 116, 232 v. Lint 227 Lobeck 47, 75 Löhlein 50, 84, 105

Longuet 214 Lopes d'Andrande 222 Lussich-Matkovich 212

M Magitot 218 Mailing 62 v. Mandach 239 Marchesani 58, 77, 93,

99, 100, no, in Márkus 304 Mawas 213

Meesmann 46, 66, 112 Meisner 62 Mettey 212, 220 Michail 330 Mironenko 247 Mita 336

Morax 213, 219 Moreau 207 Mouhiddin 249 Müller, H. K. 73, 113 Musial 189 Mylius 80, 103

N Nakayama 53 Nataf 210 Nechitsch 220 Negre 217, 250 Nida 227 Noiszewski 219

0

Ohm 395

On ken 77 d'Oswaldo 54

P

Panfique 218, 228 Papolczy 330 Passow 48, 67 Pech 213 Pesme 208 Peters 404 Petres 328

Pflimlin 89 v. Pflugk 336 Pick 120 Pillat 231 Pincus 323 Pischel 60 Poljak 245, 246, 247

412

Namenregister zu Band 72.

Pollak 124 Pollnow 120 Poos 69, 78, 399 Proksch 57

$\mathrm{R}$

Räder 53 Raubitschek 337 Redslob 211, 213, 217,

250 Rehsteiner 223 Reitsch 13, 103, 197 Renard 216, 226 Rieger 116 Riehm 91 Rifat 249 Robin

229 Roger 222

Rohrschneider 95, 97 Rollet 222 v. Rötth 115, 327 Rumjanzewa 246, 391

S Sachs 63 Safar 1, 60, 231 Sallmann 46 Salzmann 127 Samoiloff 56 Sartorius 399 Sattler 122, 243 Schäfer 398 Scheerer 98, 100 Schieck 403 Schläpfer 241, 334 Schmeichler 335

Schmelzer 57, 74 Schmidt 48, 318 Schneider 326, 334 Schreiber 77, 109 Sedan 207, 217, 224

Seidel 50 Serr 50, 51 Sexe 212 Sondermann 81 Sourdille 217 Spanlang 61 Spanuth 115 Stein, R. 332 Stewens 293, 320, 323 Stock 85 
Stoewer 52, 124, 382 Strohl 206 Stuelp 251 (P.) Süchting 32 Swerdloff 307 v. Szabó 330 v. Szily 91, 92, 93, 104

$\mathrm{T}$

Taussig 229 Terrien 211, 216, 221 Terson 222, 228 Thiel 49, 53, 55, 70, 77,

112 Thies 98, 108, 334 Thorner 81 Togano 36 Trantas 214 True 227 Tscherkes 253

$\mathrm{U}$

Ulrich 62 Ungerer 227 Urbanek 61

V Velhagen 79 Veil 217 Velter 208 Villard 213 Vincke 332 Vögele 58

Vogelsang 67, 78, 321

Vogt 54, 97. 234, 235,

236, 237, 238, 241

W Waldmann 21 Weckert 108 Weekers 229 Wegner 52, 55, 70 Weill 212 Weintraub 60 Wessely 55, 56, 75, 403 Wißmann 87 Wittelshöfer 318

$¥$

Yamanaka 58

Z. Z

Zade 108

123 Zamenhof 208 